\title{
Status of Cosmic-ray Antinuclei Searches
}

\author{
Philip von Doetinchem* \\ University of Hawaii at Manoa \\ E-mail: philipvdehawaii.edu
}

The precise measurement of cosmic-ray antiparticles and antinuclei serves as important means for identifying the nature of dark matter and other beyond-standard-model physics. Recent years showed that identifying the nature of dark matter with cosmic-ray positrons and antiprotons is difficult, and has lead to a significantly increased interest in cosmic-ray antideuteron and antihelium searches. Antideuterons and antihelium nuclei may also be generated in dark matter annihilations or decays, offering a potential breakthrough in unexplored phase space for dark matter. Low-energy antinuclei are an important approach because the flux from dark matter interactions exceeds the background flux by orders of magnitude in the low-energy range for a wide variety of models without relying on any boosting mechanisms. This talk summarizes the results of the " $2 \mathrm{nd}$ Cosmic-ray Antideuteron Workshop" that was held in March 2019 and brought together theorists and experimentalists in the field to discuss the current status, perspectives, and challenges for cosmic-ray antinuclei searches. It will review the motivation for antinuclei searches, the experimental status and its interpretation, as well as discuss some of the theoretical and experimental uncertainties of antinuclei production and propagation in our Galaxy.

36th International Cosmic Ray Conference -ICRC2019-

July 24th - August 1st, 2019

Madison, WI, U.S.A.

${ }^{*}$ Speaker. 


\section{Introduction}

The origin of dark matter is a driving question of modern physics. Low-energy antideuterons provide a "smoking gun" signature of dark matter annihilation or decay, essentially free of astrophysical background. A first-time detection of low-energy cosmic antideuterons would be an unambiguous signal of new physics, opening a transformative new field of cosmic-ray research and probing a variety of dark matter models that evade or complement collider, direct, or other cosmic-ray searches. Low-energy antiprotons are a vital partner for this analysis, and low-energy antihelium could provide further discovery space for new physics. These searches are typically referred to as indirect dark matter searches. Direct dark matter searches, i.e., dark matter scattering experiments, are most sensitive to certain couplings of dark matter to nuclei, but are mostly insensitive to other. In an era with no discovery of supersymmetry at the LHC or weakly interacting massive particles in ton-scale direct detection experiments, antinuclei signatures of dark matter have maintained their relevance in a wide variety of theories.

\section{Experiments for Antinuclei Searches}

This section gives a brief overview of current experiments sensitive to cosmic-ray antiprotons, antideuterons, and antihelium nuclei. The interpretation of the data will be reviewed in Sec. 3. The PAMELA experiment is not separately discussed in this section, but the discussion of antiproton results will also include data from PAMELA (Sec. 3.1).

\subsection{The BESS Experiment}

The BESS-Polar program exploits particle tracking in a solenoidal magnetic field to identify antimatter. The original BESS-Polar experiment flew over Antarctica in late 2004, providing the current best antideuteron flux upper limits. The BESS-Polar II experiment collected about 30 days of Antarctic flight data from December 2007 to January 2008[1, 2]. BESS-Polar II consists of a large solenoidal magnet $(0.8 \mathrm{~T})$, filled by inner drift chambers (IDC) and a jet-type drift tracking chamber (JET), and surrounded by an aerogel Cherenkov counter (ACC) and a time-of-flight system composed of scintillation counter hodoscopes. These components are arranged in a coaxial cylindrical geometry, providing a large geometric acceptance of $0.23 \mathrm{~m}^{2} \mathrm{sr}$.

Tracking is performed by fitting up to 48 hit points in the JET and 4 hit points in the IDC, resulting in a magnetic-rigidity resolution of $0.4 \%$ at $1 \mathrm{GV}$ and a maximum detectable rigidity of $240 \mathrm{GV}$. The upper and lower scintillator hodoscopes provide time-of-flight (TOF) and $\mathrm{d} E / \mathrm{d} x$ measurements as well as trigger signals. The timing resolution of each hodoscope is $120 \mathrm{ps}$, resulting in a $\beta^{-1}$ resolution of $2.5 \%$. The threshold-type Cherenkov counter, using a silica aerogel radiator with refractive index $n=1.03$, can reject electron and muon backgrounds by a factor of 12,000 . The threshold rigidities for antiproton and antideuteron are $3.8 \mathrm{GV}$ and $7.6 \mathrm{GV}$, respectively. In addition, a thin scintillator middle-TOF with timing resolution of $320 \mathrm{ps}$ is installed between the central tracker and the solenoid, in order to detect low-energy particles that cannot penetrate the magnet wall.

BESS-Polar II published antiproton results from about $200 \mathrm{MeV} / n$ up to $3 \mathrm{GeV} / n$ [1], with the smallest error bars in the range below about $500 \mathrm{MeV} / n$. Assuming that the antihelium flux would 
have the same spectral shape as the helium flux, the experiment also set an exclusion limits for antihelium in the range from $1.6-14 \mathrm{GV}$ at $1.0 \cdot 10^{-7}$ [2]. The last published result on antideuterons comes from BESS flights between 1997 and 2000 [3], which set an exclusion limit at 95\% confidence level at $1.9 \cdot 10^{-4}\left(\mathrm{~m}^{2} \mathrm{~s} \mathrm{sr} \mathrm{GeV} / n\right)^{-1}$ between 0.17 and $1.15 \mathrm{GeV} / n$. At the workshop, the BESS collaboration reported a new preliminary antiproton flux measurement based on BESS-Polar II data using the middle-TOF, which expanded the energy range down to about $120 \mathrm{MeV} n$. Also a new preliminary upper antideuteron flux limit for the BESS-Polar II flight was presented with a value of $5.9 \cdot 10^{-5}\left(\mathrm{~m}^{2} \mathrm{~s} \mathrm{sr} \mathrm{GeV} / n\right)^{-1}$ between 0.1 and $1.2 \mathrm{GeV} / n$.

\subsection{The AMS-02 Experiment}

AMS-02 is a multi-purpose cosmic-ray detector that has been operating on the International Space Station (ISS) since May 2011 [4]. It is planned to operate AMS-02 until the end of the lifetime of the ISS (at least 2024). In contrast to the high-statistics spectral measurements of other cosmic-ray species, the antideuteron and antihelium studies of AMS-02 are focused on a first-time discovery. AMS-02 follows the principle of typical magnetic spectrometer particle physics detectors, with particle identification that relies on combining signals from an array of sub-detectors.

The transition radiation detector is used to suppress particles of lower mass (like electrons, pions, and kaons). The time-of-flight (TOF) system provides the main trigger for the experiment and determines the velocity of the particle up to $\beta \approx 0.8$. The particle momentum can be extracted from its trajectory in the magnetic field $(\sim 0.15 \mathrm{~T})$. In the low-momentum region multiple scattering becomes an important effect that limits the mass resolution. In the high-velocity region two different types of ring imaging Cherenkov (RICH) counters are used (NaF, aerogel) for the velocity measurement.

The AMS-02 collaboration published the most precise antiproton flux between $1-450 \mathrm{GV}$, based on $3.49 \cdot 10^{5}$ antiproton events and $2.42 \cdot 10^{9}$ proton events [5]. Furthermore, AMS-02 announced candidate events with mass and charge consistent with antihelium [6,7]. This analysis and the antideuteron analysis are ongoing while more data is being taken.

\subsection{The GAPS Experiment}

The General Antiparticle Spectrometer (GAPS) [8] is optimized specifically for low-energy $(<0.25 \mathrm{GeV} / n)$ cosmic-ray antinuclei. The experiment consists of ten planes of semiconducting $\mathrm{Si}(\mathrm{Li})$ strip detectors surrounded on all sides by a plastic scintillator TOF. GAPS is scheduled for its first Antarctic balloon flight from McMurdo in late 2021.

GAPS relies on a novel particle identification technique based on exotic atom formation and decay [8]. First, a low-energy antiparticle that has been slowed by the atmosphere passes through the TOF system, which provides a high-speed trigger and measures particle velocity and $\mathrm{d} E / \mathrm{d} x$. It is further slowed by $\mathrm{d} E / \mathrm{d} x$ losses in the $\mathrm{Si}(\mathrm{Li})$ detectors, eventually stopping inside the detector. It then, with near unity probability, replaces a Si shell electron to form an exotic atom in an excited state. This exotic atom then de-excites through auto-ionization and radiative transitions, emitting X-rays. These X-ray energies are uniquely determined by the antiparticle and Si reduced mass and atomic numbers. Finally, the antiparticle annihilates with the Si nucleus, producing a nuclear star of pions and protons. The simultaneous occurrence in a narrow time window of Xrays of characteristic energy and nuclear annihilation products with measured multiplicity provides 
an enormously constraining signature to distinguish antiparticles and to suppress non-antiparticle background. The main challenge for identifying antideuterons is the rejection of the dominant antiproton background, but the combination of exotic atom signatures provides the required rejection.

This exotic atom detector design yields a large grasp compared to typical magnetic spectrometers, and allows for the identification of antiproton, antideuteron, and antihelium cosmic rays. GAPS will provide a precision antiproton spectrum in the low-energy range below $0.25 \mathrm{GeV} / n$. Due to the higher charge, background from antiprotons is less of a concern for the antihelium analysis, which allows for a competitive antihelium sensitivity in the low-velocity range $(\beta<0.5)$.

\section{Impact of current cosmic antinuclei measurements}

In recent years, there have been tantalizing hints of dark matter detection, for example in cosmic-ray positrons $[4,9-11]$ and $\gamma$-rays [12-19]. This section reviews the dark matter interpretation of recent antiproton measurements as well as the prospects of antideuteron and antihelium searches. It is important to note that these species need to be explained within one consistent picture. Typically, a process capable of producing heavier antinuclei will also produce antiprotons. Therefore, the antiproton flux constrains the other antinuclei fluxes when assuming certain formation antinuclei parameters. Uncertainties in the formation of antinuclei will be briefly addressed in Sec. 4.1.

\subsection{Antiproton}

The PAMELA[20, 21], BESS[1], and AMS-02[5] antiproton results have all been used to constrain dark matter models, as well as astrophysical production and propagation scenarios [22-30]. Recent studies of the AMS-02 low-energy antiproton spectrum indicate a slight excess consistent with $\sim 20-80 \mathrm{GeV}$ dark matter [31-35], invoking some of the same models indicated by the $\gamma$-ray excess from the Galactic center (Fig. ??, left). While different studies found an excess in a similar energy range, they disagree in the level of significance attributed to this finding. A recent analysis using a new technique to take into account correlations of uncertainties did not find an excess [36]. As is the case for positrons and $\gamma$-rays, also the interpretation of antiproton results requires a very good understanding of the background from standard astrophysical processes.

As an outlook for future precision measurements of the low-energy part of the antiproton spectrum with GAPS, it is important to point out that the antiproton spectrum from dark matter annihilations shifts towards lower energies with decreasing dark matter mass. Therefore, precision measurements of the very low-energy antiproton spectrum offer new phase space for probing light dark matter models [37] and primordial black holes [38-40]. Furthermore, a precision low-energy antiproton measurement is also essential to understand the propagation of all antinuclei in the Galactic and Solar environments.

\subsection{Antideuteron}

Naturally occurring antideuterons in cosmic rays from any source are predicted to have a low flux and have not yet been discovered. However, it is expected that instead of a small deviation on top of large background spectrum, dark matter annihilation or decay can produce an antideuteron signal that is orders of magnitude above the astrophysical background [22, 41-51]. 

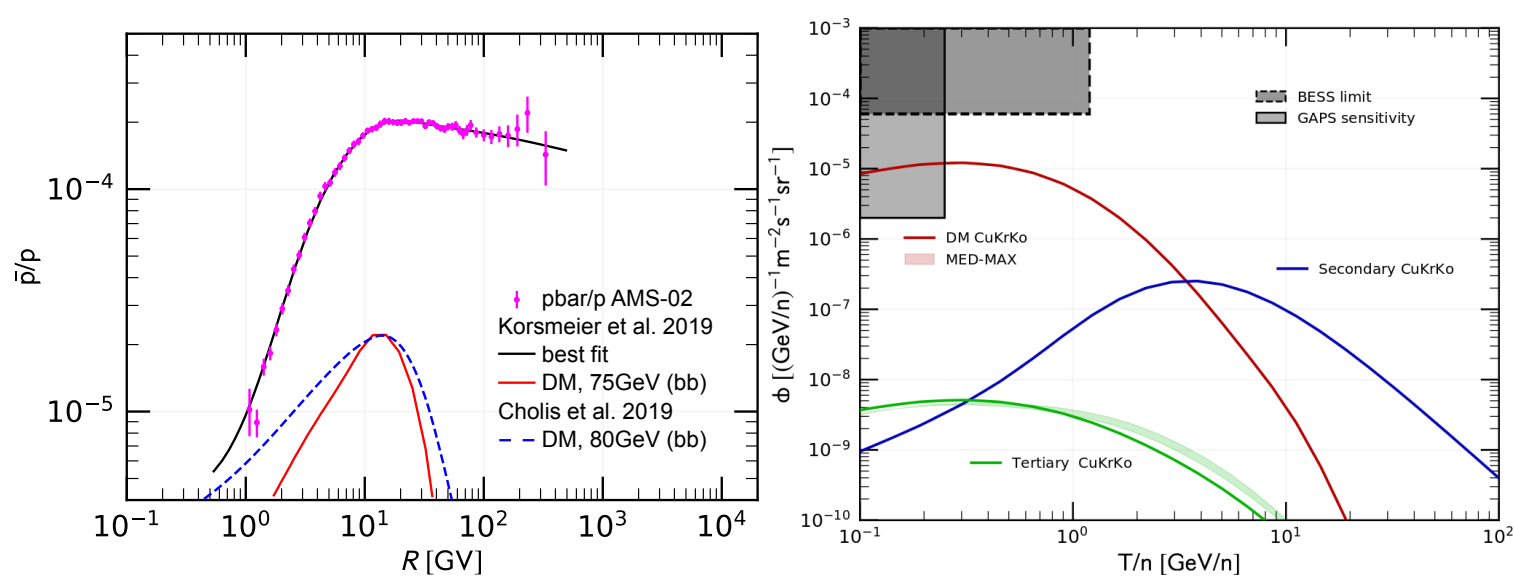

Figure 1: Left: The AMS-02 antiproton-to-proton ratio data [5] are shown together with two models invoking dark matter annihilations [34, 35]. Right: The antideuteron signal from dark matter annihilations compatible with the antiproton flux from [34] is compared to the projected sensitivities ( $3 \sigma$ discovery limit) of three 35-day GAPS flights [8] and the new limit from BESS-Polar II.

Secondary/tertiary (background) antideuterons are produced when cosmic-ray protons or antiprotons interact with the interstellar medium $[44,46]$. The high energy threshold for antideuteron production and the steep energy spectrum of cosmic rays mean that there are few particles with sufficient energy to produce secondary antideuterons, and those that are produced have relatively large kinetic energy. The right side of Fig. 1 illustrates the importance of low-energy antideuteron searches: the antideuteron flux from generic dark matter annihilations $\left(m_{\mathrm{DM}}=71 \mathrm{GeV}\right)$ into $b \bar{b}$ pairs [48]) followed by hadronization and coalescence exceeds the astrophysical background by more than two orders of magnitude in the energy range below a few $\mathrm{GeV} / n$.

Antideuterons are sensitive to a broad range of specific dark matter candidates, e.g. a lightest supersymmetric particle (LSP) neutralino [43], a right-handed Kaluza-Klein neutrino of extradimensional grand unified theories (LZP) [42], a decaying LSP gravitino [47], a dark matter particle with gluonic decay channels [52], or candidates in the next-to-minimal supersymmetric model (NMSSM) [26]. Depending on details for antideuteron formation and cosmic-ray propagation models, the experimental sensitivities reach well below the thermal relic cross section for annihilation into light quarks for dark matter with mass below 20-100 GeV [22]. A detectable antideuteron signal may also result from annihilation in certain heavy $(5-20 \mathrm{TeV})$ WIMP models, if one assumes a cosmic-ray propagation scenario with a larger halo size, as favored by AMS-02 antiproton data [53]. In addition, models that combine TeV-mass, pure-Wino dark matter with Sommerfeld enhancement mechanisms can result in accessible antideuteron signals [54]. Heavy dark matter models are particularly challenging for direct detection and collider methods.

\subsection{Antihelium}

Antihelium nuclei reaching Earth's vicinity from antimatter-dominated regions of the universe are nearly excluded $[55,56]$. This is particularly interesting in the light of the announcement by the AMS-02 collaboration of antihelium candidate events [6,7]. Data taking, analyses, and interpretation are ongoing. The interpretation spans a range from modified antihelium formation 
models [49, 57], dark matter annihilations [48, 51, 58-60], or the postulation of the existence of an antistar in our Galaxy [61]. The confirmation of any cosmic-ray antihelium would be transformative and refashion the field of cosmic-ray physics and potentially revolutionize the understanding of Big Bang nucleosynthesis.

\section{Uncertainties for cosmic antinuclei flux predictions}

The relative theoretical uncertainties of antideuteron formation and cosmic-ray propagation are both on the order of 10 [62]. These uncertainties can be reduced with cross section measurements at ground-based experiments.

\subsection{Formation}

The hadronic antinuclei formation process breaks the degeneracy of antinuclei with antiprotons and is described by different models, which are not well understood [63]. In the coalescence model, antideuterons can be formed by an $p-n$ pair if their relative momentum is small compared to the coalescence momentum $p_{0}$. Extracting $p_{0}$ from fits to available data leads to the coalescence momentum describing not only the momentum difference of the constituents, but also all other underlying conditions that lead to antinuclei formation. In the thermal model, antinuclei are directly formed at the hadronization stage, without producing separate antinucleons first. Recently there has been also more activity to include a more complete quantum mechanical description of the formation process [57, 64, 65]. While more experimental data at high energies from ALICE become available [66], more experimental data on antinuclei formation at lower energies are needed.

\subsection{Propagation}

An important constraint for antinuclei flux from dark matter annihilations is the Galactic halo size, which directly scales the observable flux [67]. Fits of cosmic-ray nuclei data are very important to constrain cosmic-ray propagation models (e.g., Li/C, Li/O, Be/C, Be/O, B/C, B/O) [33, 36]. Some of these studies are restricted by the precision and availability of cross section measurements of relevant primary cosmic ray species with the the interstellar medium [68].

\section{The Coming Decade and Beyond}

Cosmic-ray antinuclei searches are currently undergoing a transformation. For a long time, antiproton results were used to provide constraints for heavier antinuclei species. The potential detection of antihelium events is posing the question of the underlying production mechanism, which should explain the antihelium events and at the same time not overproduce antiprotons nor antideuterons. Hence, the coming decade is an exciting time for experimental searches for cosmicray antinuclei. Experiments are beginning to be sensitive to viable dark matter models, and thus the lifetimes of current missions must be supported and future mission technologies and concepts must be developed in parallel. Within the AMS-02 program, antinuclei searches are among the measurements that will significantly benefit from extended data taking on the ISS. The ultimate goal of the GAPS program is three 35-day long duration balloon flights. The current experiments sensitive to cosmic-ray antinuclei can be seen as discovery experiments. Successor experiments 
will need to aim at measuring spectra with high statistics. This is only possible with extended data taking and larger payloads, e.g., on the Moon's surface or at one of the Lagrange points.

\section{Acknowledgments}

The second dedicated cosmic-ray antideuteron workshop at UCLA in March 2019 was supported, in part, by the University of California, Los Angeles. The work was supported by the National Science Foundation under Award No. 1551980. The author would like to thank all workshop participants and organizers.

\section{References}

[1] Abe, K. et al. Physical Review Letters 108, 051102 (2012).

[2] Abe, K. et al. Physical Review Letters 108, 131301 (2012).

[3] Fuke, H. et al. Physical Review Letters 95, 081101 (2005).

[4] Accardo, L. et al. Physical Review Letters 113, 121101 (2014).

[5] Aguilar, M. et al. Physical Review Letters 117, 091103 (2016).

[6] Ting, S. Press Conference at CERN, December 8 (2016).

[7] Ting, S. Colloquium at CERN, May 24 (2018).

[8] Aramaki, T. et al. Astroparticle Physics 74, 6-13 (2016).

[9] Aguilar, M. et al. Physical Review Letters 110, 141102 (2013).

[10] Aguilar, M. et al. Physical Review Letters 122, 041102 (2019).

[11] Abeysekara, A. U. et al. Science 358, 911-914 (2017).

[12] Hooper, D. \& Linden, T. Physical Review D 84, 123005 (2011).

[13] Daylan, T. et al. Physics of the Dark Universe 12, 1-23 (2016).

[14] Kaplinghat, M., Linden, T. \& Yu, H.-B. Physical Review Letters 114, 211303 (2015).

[15] Achterberg, A. et al. J. Cosmol. Astropart. P. 8, 6 (2015).

[16] Ajello, M. et al. Astrophysical Journal 819, 44 (2016).

[17] Calore, F., Cholis, I., McCabe, C. \& Weniger, C. Physical Review D 91, 063003 (2015).

[18] Ackermann, M. et al. Astrophys. J. 840, 43 (2017).

[19] Hooper, D. \& Linden, T. Physical Review D 98, 043005 (2018).

[20] Adriani, O. et al. Physical Review Letters 105, 121101 (2010).

[21] Adriani, O. et al. JETP 96, 621-627 (2013).

[22] Fornengo, N., Maccione, L. \& Vittino, A. J. Cosmol. Astropart. P. 1309, 031 (2013).

[23] Cirelli, M. \& Giesen, G. J. Cosmol. Astropart. P. 1304, 015 (2013).

[24] Hooper, D., Linden, T. \& Mertsch, P. J. Cosmol. Astropart. P. 1503, 021 (2015).

[25] Boudaud, M., Cirelli, M., Giesen, G. \& Salati, P. J. Cosmol. Astropart. P. 5, 13 (2015).

[26] Cerdeño, D., Peiró, M. \& Robles, S. J. Cosmol. Astropart. P. 1408, 005 (2014).

[27] Hamaguchi, K., Moroi, T. \& Nakayama, K. Physics Letters B 747, 523-528 (2015).

[28] Kappl, R., Reinert, A. \& Winkler, M. W. J. Cosmol. Astropart. P. 10, 034 (2015).

[29] Chen, C.-H., Chiang, C.-W. \& Nomura, T. Physics Letters B 747, 495-499 (2015).

[30] Winkler, M. W. J. Cosmol. Astropart. P. 1702, 048 (2017).

[31] Cui, M.-Y., Yuan, Q., Tsai, Y.-L. S. \& Fan, Y.-Z. Physical Review Letters 118, 191101 (2017). 
[32] Cuoco, A., Krämer, M. \& Korsmeier, M. Physical Review Letters 118, 191102 (2017).

[33] Reinert, A. \& Winkler, M. W. J. Cosmol. Astropart. P. 1801, 055 (2018).

[34] Cuoco, A., Heisig, J., Klamt, L., Korsmeier, M. \& Krämer, M. (2019).

[35] Cholis, I., Linden, T. \& Hooper, D. Physical Review D 99, 103026 (2019).

[36] Boudaud, M. et al. arXiv:1906.07119 (2019).

[37] Aramaki, T. et al. Astroparticle Physics 59, 12-17 (2014).

[38] Page, D. N. \& Hawking, S. Astrophysical Journal 206, 1-7 (1976).

[39] Maki, K., Mitsui, T. \& Orito, S. Physical Review Letters 76, 3474-3477 (1996).

[40] Barrau, A. et al. Astronomy and Astrophysics 388, 676-687 (2002).

[41] Donato, F., Fornengo, N. \& Salati, P. Physical Review D 62, 043003 (2000).

[42] Baer, H. \& Profumo, S. J. Cosmol. Astropart. P. 0512, 008 (2005).

[43] Donato, F., Fornengo, N. \& Maurin, D. Physical Review D 78, 043506 (2008).

[44] Duperray, R. et al. Physical Review D 71, 083013 (2005).

[45] Ibarra, A. \& Wild, S. J. Cosmol. Astropart. P. 1302, 021 (2013).

[46] Ibarra, A. \& Wild, S. Physical Review D 88, 023014 (2013).

[47] Dal, L. \& Raklev, A. Physical Review D 89, 103504 (2014).

[48] Korsmeier, M., Donato, F. \& Fornengo, N. Physical Review D 97, 103011 (2018).

[49] Tomassetti, N. \& Oliva, A. Proceedings of Science EPS-HEP2017, 620 (2017).

[50] Lin, S.-J., Bi, X.-J. \& Yin, P.-F. arXiv:1801.00997 (2018).

[51] Ding, Y.-C., Li, N., Wei, C.-C., Wu, Y.-L. \& Zhou, Y.-F. J. Cosmol. Astropart. P. 1906, 004 (2019).

[52] Cui, Y., Mason, J. D. \& Randall, L. Journal of High Energy Physics 1011, 017 (2010).

[53] Bräuninger, C. B. \& Cirelli, M. Physics Letters B 678, 20-31 (2009).

[54] Hryczuk, A., Cholis, I., Iengo, R., Tavakoli, M. \& Ullio, P. J. Cosmol. Astropart. P. 1407, 031 (2014).

[55] Adams, F. C., Freese, K., Laughlin, G., Tarle, G. \& Schwadron, N. Astrophysical Journal 491, 6-12 (1997).

[56] Cohen, A. G., De Rujula, A. \& Glashow, S. Astrophysical Journal 495, 539-549 (1998).

[57] Blum, K., Ng, K. C. Y., Sato, R. \& Takimoto, M. Physical Review D 96, 103021 (2017).

[58] Cirelli, M., Fornengo, N., Taoso, M. \& Vittino, A. Journal of High Energy Physics 1408, 009 (2014).

[59] Carlson, E. et al. Physical Review D 89, 076005 (2014).

[60] Coogan, A. \& Profumo, S. Physical Review D 96, 083020 (2017).

[61] Poulin, V., Salati, P., Cholis, I., Kamionkowski, M. \& Silk, J. Physical Review D 99, 023016 (2019).

[62] Aramaki, T. et al. Physics Reports 618, 1-37 (2016).

[63] Gomez-Coral, D.-M. et al. Physical Review D 98, 023012 (2018).

[64] Scheibl, R. \& Heinz, U. W. Physical Review C 59, 1585-1602 (1999).

[65] Kachelrieß, M., Ostapchenko, S. \& Tjemsland, J. arXiv:1905.01192 (2019).

[66] Acharya, S. et al. Phys. Rev. C97, 024615 (2018).

[67] Donato, F., Fornengo, N., Maurin, D. \& Salati, P. Physical Review D 69, 063501 (2004).

[68] Genolini, Y., Maurin, D., Moskalenko, I. V. \& Unger, M. Physical Review C 98, 034611 (2018). 